\title{
45,X/46,XY mixed gonadal dysgenesis: A case of successful sperm extraction
}

\author{
Ryan Kendrick Flannigan, MD; ${ }^{*}$ Victor Chow, MD FRCSC; ${ }^{+}$Sai Ma, PhD; ${ }^{\ddagger}$ Albert Yuzpe, MD, MSc, FRCSC ${ }^{\dagger}$
}

*Department of Urological Sciences, University of British Columbia, Vancouver, BC; †Department of Obstetrics and Gynecology, Department of Urological Sciences, Genesis Fertility Clinic, University of British Columbia, Vancouver, BC; §Division of Reproductive Endocrinology and Infertility, Department of Obstetrics and Gynecology, University of British Columbia, Vancouver, BC

Cite as: Can Urol Assoc J 2014;8(1-2):e108-10. http://dx.doi.org/10.5489/cuaj.1574 Published online February 12, 2014.

\section{Abstract}

Infertility is common among couples, about one third of cases are the result of solely male factors, and rarely abnormalities of genetic karyotypes are the root cause. Individuals with a $45 \mathrm{X}, / 46, \mathrm{XY}$ mosaiscism are rare in the literature and very few have fertile potential. We discuss a case of a 27-year-old male with known mixed gonadal dysgenesis, 50:50 split mosaiscism of 45,X/46,XY, presenting for evaluation of 1.5 year history of infertility. He demonstrated low volume non-obstructive azoospermia. Upon testicular biopsy, spermatozoa were extracted. These sperm were subjected to aneuploidy studies demonstrating $95.95 \%$ euploidy. The sperm were further assessed and placed in cryopreservation after being deemed sufficient for potential intracytoplasmic sperm injection. This is a unique case of viable sperm in a man with mixed gonadal dysgenesis, 45,X/46, XY mosaiscism.

\section{Introduction}

Sohval in 1963 first described mixed gonadal dysgenesis (MGD). ${ }^{1}$ Most patients have a 45,X/46, XY karyotype, and have an abnormal testis with contralateral streak gonad. ${ }^{2}$ This is thought to occur via chromosomal misaggregation secondary to anaphase lag or chromosomal rearrangement during early embryonic mitosis. ${ }^{3}$ The phenotypic abnormalities are the result of incomplete inhibition of mullerian structures, incomplete mesonephric duct structure differentiation and incomplete masculanization of external genitalia. ${ }^{4}$ In fact, in one series, 95\% of MGD patients had mullerian remnants and $75 \%$ of streak gonads had an ipsilateral fallopian tube. ${ }^{4}$ Therefore, it is obvious that individuals with $45, X / 46, X Y$ karyotype and MGD may present with a variable phenotype.

Individuals with this phenotype often present as children with ambiguous or abnormal genitalia, or as adults with gonadal failure or short stature..$^{5-7}$ Associated characteristics include cardio renal malformations, gonadal blastomas and germ cell tumours. The phenotype of these patients tends to vary, but can be somewhat predicted based upon location and extent of gonadal development. Layman and colleagues ${ }^{4}$ and Gantt and collegues ${ }^{6}$ suggest that those individuals with bilateral streaks are associated with the phenotype of a sexually infantile female; those with a streak and intra-abdominal testis present with clitoromegaly in a female; individuals with one scrotal testis and an intra abdominal streak are associated with frank sexual ambiguity and bilateral scrotal testis tends to present as a male with short stature and gonadal failure.

Infertility, not amenable to treatment, is the final analysis with these patients; however, some have explored intracytoplasmic sperm injection (ICSI) in rare cases of MGD with spermatogonia present. ${ }^{8}$ Testicular pathologic investigations typically reveal the presence of mature seminiferous tubules; however, the architecture is typically disorganized with hyalinization and atrophy of the tubules. ${ }^{8}$ In a recent case series of 20 MGD patients, none have been successfully fertile. Of these patients, $45 \%$ developed testicular failure necessitating testosterone replacement. ${ }^{9}$ Among this cohort, $63 \%$ of patients were had $\mathrm{Y}$ chromosomal rearrangements that had previously been documented to impair fertility. ${ }^{10-12}$ Arnedo and colleagues described an individual with a 45,X/46,X(r) Y karyotype, phenotypically healthy adult male with oligozoospermia. ${ }^{13}$ This MGD patient was able to conceive with his wife; they had a son with Klinefelter syndrome and a karyotype of $47, \mathrm{XX}, \mathrm{r}(\mathrm{Y}) / 46, \mathrm{XX}$. Bofinger and colleagues described successful conception of offspring with use of ICSI in the setting of $45, X / 46, X(r) Y$ karyotypes. ${ }^{14}$ This individual presented with oligoasthenoteratozoospermia. Additionally, Blanco and colleagues described a patient also presenting with oligozoospermia that was subsequently analyzed. ${ }^{15}$ Thus, most patients with MGD are infertile with very few reports of spermatagonia and even fewer reports of successful conception. 


\section{Clinical case}

A 27-year-old male presented with his 25-year-old female partner for infertility assessment. The couple had been using unprotected intercourse for about about 1.5 years without a successful pregnancy. The female had been investigated for fertility potential with no abnormalities identified.

The male patient's medical history is significant for MGD diagnosed as an infant; specifically, his karyotype is a mosaicism of a 50:50 split 46,XY/45,X. He initially presented with a penoscrotal hypospadias and bifid scrotum. This was corrected surgically throughout his childhood with a two-stage hypospadias repair. The result is a coronal meatus. He also has had a right orchiectomy with pathology demonstrating dysgenesis. He also had a prostatic utricle and concomitant urinary tract infections. As such, at age 2, the utricle was excised and over sewn through an open cystotomy. The structure was grossly mucosal and no adherence to adjacent structures was present. Screening infantile abdominal ultrasound demonstrated normal kidneys bilaterally with no ovarian structures. Growth during infancy and childhood was at the $5^{\text {th }}$ percentile; however, bone age was 1.5 years greater than true age leading up to puberty. He is otherwise healthy, aside from a diagnosis of psoriasis. No additional risk factors or family history were evident upon further questioning.

Physical examination revealed a healthy appearing, hemodynamically stable man. He was well-virilized with no gynecomastia. His abdominal examination was benign. External genital examination revealed a solitary left testicle, about $20 \mathrm{cc}$ in size, with a vas deferens palpable and no obvious varicocele. Laboratory investigations demonstrated a normal complete blood count, electrolytes and creatinine. However, his follicle-stimulating hormone was elevated at $14 \mathrm{IU} / \mathrm{L}$, luteinizing hormone was normal at $8.3 \mathrm{IU} / \mathrm{L}$ and total testosterone was also normal at $13 \mathrm{nmol} / \mathrm{L}$. The patient's genome was also analyzed for a Y chromosome microdeletion. The result of this test was negative for microdeletions in regions AZFa, AZFb and AZFC.

On history, his erectile function and ejaculation were normal. However, he and his partner had been unable to conceive after 18 months of unprotected intercourse. Subsequently, both partners were investigated for infertility. Two semen analyses were acquired; they demonstrated low volume, 0.2 to $0.4 \mathrm{~mL}$, azoospermia.

A testicular biopsy was performed to acquire a cytogenetic analysis and to assess for the presence of sperm. The procedure revealed a yellow-brown discoloration of the seminiferous tubules. Three samples were collected. The first was placed in a sperm buffer and sent for potential sperm cryopreservation. The second was to be sent for genetic analysis and the third was placed in a buffered zinc formalin for pathology.
The pathologist reported the presence of spermatogenesis, thickening of the basement membrane and prominent Leydig cell hyperplasia. This report was in keeping with the clinical history of MGD. The cryopreservation semen analysis of the testicular biopsy isolated 103 sperm in the pre-thaw analysis. Of these, 7 were non-progressive and 96 were immotile. In the post-thaw analysis, 2 were non-progressive and 621 immotile; with the addition of pentoxyfiline, 1 was slow progressive, 24 non-progressive and 310 immotile. The interpretation of the report indicated that the cryopreserved samples of sperm would be suitable for ICSI.

The remaining sample from the biopsy was sent for a fluorescence in situ hybridization assay (FISH) assessment for sperm aneuploidy. A total of 1702 sperm were scored. Of these, $95.95 \%$ of the sperm were euploid with $63.57 \% 18 \mathrm{X}$ and $32.37 \% 18 \mathrm{Y}$. The remaining $4.05 \%$ were aneuploidy; more specifically, $0.18 \%$ were disomy $18,2.12 \%$ were sex disomy, $1.65 \%$ were sex nullisomy, and $0.18 \%$ diploid.

The patient and his partner proceeded for a round of ICSI. The first round was unsuccessful; in this cycle, 12 oocytes were retrieved, 11 were inseminated, 9 embryos were obtained and 2 were deemed as satisfactory for transfer; however, no embryos were successful by day 3 . They subsequently underwent a second round of ICSI with half eggs fertilized with a donor and half with the patient's sperm to optimize success of the cycle, at the recommendation of the fertility clinic. In total, 8 oocytes were collected, 7 were inseminated with 3 via the patient's sperm and 4 via donor sperm. No embryos were viable from the patient, and only 2 embryos were transferred from the donor, with 1 viable pregnancy.

\section{Methods}

\section{$Y$ chromosome microdeletion analysis}

DNA was acquired from peripheral lymphocytes. The DNA was then subjected to polymerase chain reaction (PCR) and assayed using the following tagged sequences: ZFY (control), sy14 (SRY); AZFa specifically, sy84, sy86, sy625, sy114; AZFb specifically, sy117, sy127, sy129, sy134, sy143; AZFc specifically, sy 152 , sy 147 , sy 254 , sy 255 , sy 157 ; and finally heterochromatin sy160.

\section{FISH analysis}

A FISH analysis was performed on the testicular spermatozoa. Aneu Vysion Multicolor DNA Probe Kit, (Vysis Inc., Downers Grobe, IL) consisting of a probe mixture of CEP 18, CEP $X, C E P Y$ was used to assess the spermatozoa. 


\section{Sperm analysis}

A testicular biopsy was performed and placed in a sperm biopsy. The tissue was shredded and centrifuged down to $0.5 \mathrm{~mL}$. In total, $5 \mathrm{ul}$ was aspirated from the sample and added to the whole Makler grid to assess motility; 2 vials of the $0.5-\mathrm{mL}$ samples were frozen, then thawed for a postthaw analysis. One sample was assessed for pentoxifylline and one in the absence of pentoxifylline. The remaining sample was frozen for potential future use.

\section{Conclusion}

Cases of mosaic $46, X Y / 45, X$ males have been described. The phenotypes of these individuals tend to vary, but often present as infertility, ambiguous genitalia or short stature. There have been very limited reports of fertility in these patients. Our patient had a 50:50 mosaic 46,XY/45,X with azoospermia; he was amenable to testicular sperm extraction and ICSI-assisted fertility. In this case, ICSI was not successful. It was felt that this was likely related to the failure of ICSI, and not a sperm factor, since the sperm on testicular biopsy demonstrated potential for successful ICSI and thus conception. Future attempts of ICSI with this individual's sperm would not be precluded nor should others with similar karyotypes. This cohort of individuals should receive the warranted investigations and opportunity for sperm extraction and ICSI if desired, as fertility may be possible.

Competing interests: Dr. Flannigan, Dr. Chow, Dr. Ma and Dr. Yuzpe all declare no competing financial or personal interests.

This paper has been peer-reviewed.

\section{References}

1. Sohval AR. Mixed gonadal dysgenesis: A variety of hermaphroditism. Am J Hum Genet 1963;15:155.

2. Brosman SA. Mixed gonadal dysgenesis. J Urol 1979;121:344.

3. Hsu LY. Phenotype/karyotype correlations of $Y$ chromosome aneuploidy with emphasis on structural aberrations in postnatally diagnosed cases. Am J Med Genet 1994;53:108-40. http://dx.doi.org/10.1002/ ajmg.1320530204

4. Robboy SJ, Miller T, Donahoe PK, et al. Dysgenesis of testicular and streak gonads in the syndrome of mized gonadal dysgenesis: Perspective Derived from a Clinicopathologic Analysis of Twenty-one Cases. Hum Pathol 1982;13:700-16. http://dx.doi.org/10.1016/S0046-8177(82)80292-X

5. Layman LC, Tho SPT, Clark A, et al. Phenotypic spectrum of $45, X / 46, X Y$ males with a ring $Y$ chromosome and bilaterally descended testes. Fertil Steril 2009;91:791-7. http://dx.doi.org/10.1016/j. fertnstert.2007.12.078

6. Gantt PA, Byrd JR, Greenblatt RB, et al. A clinical and cytogenetic study of fifteen patients with $45, X / 46 X Y$ gonadal dysgenesis. Fertil Steril 1980;34:216-21.

7. Telvi L, Lebbar A, Del Pino 0, et al. 45,X/46,XY mo- saicism: Report of 27 cases. Pediatrics 1999; 104:3048. http://dx.doi.org/10.1542/peds.104.2.304

8. Hsieh MH, Hollander A, Lamb DJ, et al. The genetic and phenotypic basis of infertility in men with pediatric urologic disorders. J Urol 2010;76:25-31. http://dx.doi.org/10.1016/i.urology.2010.03.011

9. Martinerie L, Morel Y, Gay C, et al. Impaired puberty, fertility and final stature in $45, X / 46, X Y$ mixed gonadal dysgenetic patients raised as boys. Eur J Endocrinol 2012;166:687-94. http://dx.doi. org/10.1530/EJE-11-0756

10. Tiepolo L, Zuffardi 0 . Localization of factors controlling spermatogenesis in the nonfluorescent portion of the human $Y$ chromosome long arm. Hum Genet 1976;34:119-24. http://dx.doi.org/10.1007/ BF00278879

11. Vogt PH, Edelmann A, Kirsch S, et al. Human $Y$ choromosome azoospermia factors (AZF) mapped to different subregions in Yq1 1. Hum Mole Genet 1996;5:933-43. http://dx.doi.org/10.1093/hmg/5.7.933

12. Roberts KP. Y-chromosome deletions and male infertility: State of the art and clinical implications. I Androl 1998; 19:255-9.

13. Arnedo $N$, Nogues $C$, Bosch $M$, et al. Mitotic and meiotic behaviour of a naturally transmitted ring $Y$ chromosome: Reproductive risk evaluation. Hum Reprod 2005;20:462-8. http://dx.doi.org/10.1093/ humrep/deh598

14. Bofinger MK, Needham DF, Saldana $L R$, et al. $45, X / 46, X, r(Y)$ karyotype transmitted by father to son after intracytoplasmic sperm injection for oligospermia. J Reprod Med 1999;44:645-8.

15. Blanco J, Farreras A, Egozcue J, et al. Meiotic behaviour of the sex chromosomes in a 45,X/46,X,r(Y)/46,X,dic r(Y) patient assessed by FISH. Fertil Steril 2003;79:913-8. http://dx.doi. org/10.1016/S0015-0282(02)04931-2

Correspondence: Dr. Ryan Kendrick Flannigan, Department of Urological Sciences, University of British Columbia, Vancouver, BC; rkflanni@gmail.com 